\title{
BMJ Open Disability acquisition and mental health: effect modification by demographic and socioeconomic characteristics using data from an Australian longitudinal study
}

Zoe Aitken, ${ }^{1}$ Julie Anne Simpson, ${ }^{2}$ Rebecca Bentley, ${ }^{1}$ Anne Marie Kavanagh ${ }^{1}$

To cite: Aitken Z, Simpson JA, Bentley R, et al. Disability acquisition and mental health: effect modification by demographic and socioeconomic characteristics using data from an Australian longitudinal study. BMJ Open 2017;7:e016953. doi:10.1136/ bmjopen-2017-016953

- Prepublication history and additional material are available. To view these files please visit the journal online (http://dx.doi org/10.1136/bmjopen-2017016953).

Received 22 March 2017 Revised 14 June 2017 Accepted 13 July 2017

\section{(a) CrossMark}

${ }^{1}$ Gender and Women's Health Unit, Centre for Health Equity, Melbourne School of Population and Global Health, The University of Melbourne, Carlton, Australia ${ }^{2}$ Biostatistics Unit, Centre for Epidemiology and Biostatistics, Melbourne School of Population and Global Health, The University of Melbourne, Carlton, Australia

Correspondence to

Dr Zoe Aitken;

zoe.aitken@unimelb.edu.au

\section{ABSTRACT}

Objectives There is evidence of a causal relationship between disability acquisition and poor mental health, but the substantial heterogeneity in the magnitude of the effect is poorly understood and may be aetiologically informative. This study aimed to identify demographic and socioeconomic factors that modify the effect of disability acquisition on mental health.

Design and setting The Household, Income and Labour Dynamics in Australia Survey is a nationally representative Iongitudinal survey of Australian households that has been conducted annually since 2001. Four waves of data were included in this analysis, from 2011 to 2014.

Participants Individuals who acquired a disability $(n=387)$ were compared with those who remained disability-free in all four waves $(n=7936)$.

Primary outcome measure Mental health was measured using the mental health subscale of the Short Form 36 (SF-36) general health questionnaire, which measures symptoms of depression, anxiety and psychological wellbeing.

Methods Linear regression models were fitted to estimate the effect of disability acquisition on mental health, testing for effect modification by key demographic and socioeconomic characteristics. To maximise causal inference, we used a propensity score approach with inverse probability of treatment weighting to control for confounding and multiple imputation using chained equations to assess the impact of missing data.

Results On average, disability acquisition was associated with a 5-point decline in mental health score (estimated mean difference: $-5.1,95 \% \mathrm{Cl}-7.2$ to -3.0). There was strong evidence that income and relationship status modified the effect, with more detrimental effects in the lowest $(-12.5,95 \% \mathrm{Cl}-18.5$ to -6.5$)$ compared with highest income quintile $(-1.1$, $95 \% \mathrm{Cl}-4.9$ to 2.7 ) and for people not in a relationship $(-8.8,95 \% \mathrm{Cl}-12.9$ to -4.8$)$ compared with those who were $(-3.7,95 \% \mathrm{Cl}-6.1$ to -1.4$)$.

Conclusions Our results suggest that the detrimental effect of disability acquisition on mental health is substantially greater for socioeconomic disadvantaged individuals.
Strengths and limitations of this study

- This study uses data from a large nationally representative longitudinal study in Australia to model the effect of disability acquisition on mental health and identify demographic and socioeconomic factors that modify the association.

- This is the first study to quantify mental health inequalities associated with a comprehensive range of demographic and socioeconomic factors to better understand the heterogeneity in the mental health inequalities experienced by people with disabilities.

- To maximise causal inference, we employed propensity score models with inverse probability weighting to better control for confounding and multiple imputation to assess the impact of missing data on the results.

- Limitations include the use of self-reported data, missing data (although multiple imputation was used) and underrepresentation of people with severe disabilities.

- The finding that the detrimental effect of disability acquisition on mental health is substantially greater for socioeconomically disadvantaged individuals has important implications for disability, social and public health policies, as it identifies particularly vulnerable subgroups of people with disabilities who are likely to experience greater mental health effects.

\section{INTRODUCTION}

Currently, one in five Australians has a disability. ${ }^{1}$ People with disabilities experience substantial health inequalities compared with those without disabilities, reporting poorer health across a wide range of indicators including mental health. ${ }^{2}$ For example, in Australia, a large survey found that $48 \%$ of people reporting severe disabilities experienced mental health problems compared with $6 \%$ of those without disabilities. ${ }^{4}$

Evidence from longitudinal studies has demonstrated that disability acquisition is 
associated with a deterioration in mental health, ${ }^{2}{ }^{35-10}$ suggesting a causal relationship between disability and poor mental health. However, not all people who acquire a disability experience a decline in their mental health, ${ }^{11}$ there is substantial heterogeneity in the magnitude of the association. Understanding this heterogeneity may be aetiologically informative and may shed light on the structural drivers of the inequalities. ${ }^{12}{ }^{13}$ Intersectionality theory has been posited as a way to understand how social identities (eg, gender) and positions (eg, socioeconomic disadvantage) interact to shape people's experiences. ${ }^{14}$ This approach suggests that social determinants of health cannot be understood independently; rather the focus should be on understanding how factors intersect and mutually reinforce each other in their health impacts. ${ }^{15}$ Bauer argues that intersectionality theory can be operationalised in epidemiological studies by fitting interactions between different exposure variables. ${ }^{14}$ In this study, we explore how demographic, social and economic factors interact with disability to modify its effect on mental health.

There is limited evidence about how the association between disability and mental health varies according to demographic and socioeconomic characteristics. Four longitudinal studies have examined whether the association between disability acquisition and mental health differs according to socioeconomic characteristics. Two studies of working aged Australians found evidence that the association varied according to housing characteristics and wealth; the largest mental health declines were seen for people in unaffordable and insecure housing ${ }^{2}$ and with low wealth. ${ }^{3}$ Likewise, a study of people approaching retirement age in the USA found that the negative effect of disability on subjective well-being was greater in individuals with low wealth prior to disability acquisition. ${ }^{6}$ Finally, a study of individuals aged 33 years from the UK found larger effects of disability acquisition on psychological distress for people with low education. ${ }^{5}$ None of the studies comprehensively examined a range of factors that influence the effect of disability acquisition on mental health to fully understand important demographic and socioeconomic determinants and their relative importance.

A better understanding of the characteristics that determine the magnitude of the mental health effects for people who acquire a disability will provide information that can be used to inform the development of targeted social and health policies for people with disabilities most likely to experience poorer mental health. Such interventions are likely to improve the mental health of people with disabilities, but may also have implications for longterm health and welfare costs associated with disability. This analysis uses data from a longitudinal study to model relationships between disability acquisition and mental health, testing for effect modification by demographic and socioeconomic characteristics prior to disability and quantifying excess mental health effects associated with these characteristics.

\section{METHODS}

\section{Data source}

The Household, Income and Labour Dynamics in Australia (HILDA) Survey is a longitudinal study of Australian households, conducted annually since 2001. The survey collects information about the demographic, social, economic and health characteristics of individuals using a combination of interviews and self-completion questionnaires. Data are collected on all household members, with interviews conducted with those aged 15 years and older. The original sample included 13969 participants from 7682 households, randomly sampled using a national probability sample of private dwellings. In later waves, continuing survey members included all participants from the original sample, any children born or adopted in the household and new partners. Additional households were added in later waves to maintain representativeness, with a sample size after 14 waves of 28794 people. Response rates were above $70 \%$ for new participants and above $90 \%$ for continuing respondents. Full details of HILDA are available elsewhere. ${ }^{16}$

\section{Disability acquisition}

Information about disability was collected from participants in every wave, defined in HILDA as 'a long-term health condition, impairment or disability that restricts you in your everyday activities, and has lasted or is likely to last for 6 months or more'. If participants reported a disability, they were then asked to describe the type of impairment, such as limited use of fingers or arms, or problems with eyesight that could not be corrected with glasses or contact lenses. Different impairment types were not examined separately because we lacked power to examine differences by disability characteristics but also to make the results relevant to disability policies which generally do not address different types of impairments specifically. The analysis was restricted to the most recent four waves of the survey (2011-2014) to enable selection of comparable groups to maximise exchangeability. Participants were included in the analysis if they were disability-free for the first two consecutive waves of the analysis followed by either two consecutive waves of disability (disability group) or two consecutive waves of no disability (control group). We used two consecutive waves of disability so as to exclude people with transient disability and to reduce the potential for measurement error. Eligibility for inclusion required participation in all four waves and response to the disability question at every wave.

\section{Mental health}

The Short Form 36 is a widely used general health questionnaire that has been validated for use in the Australian population. ${ }^{17}$ It comprises eight subscales measuring various components of health and two overall summary measures. The Mental Health Inventory (MHI), one of the subscales, is composed of five items relating to mental health over the previous 4 weeks, specifically measuring 
symptoms of depression, anxiety and psychological wellbeing. Each item is scored using five response categories, and the total scores are transformed into a scale ranging from 0 to 100, with higher scores reflecting better mental health.

\section{Covariates}

Covariates were measured in the first wave contributing to the analysis, as a measure of circumstances prior to disability acquisition. Demographic characteristics consisted of age, sex and country of birth. Socioeconomic characteristics included education, employment, income (population quintiles of equivalised household disposable income), financial hardship, housing tenure, housing affordability (unaffordable defined as households in the lowest $40 \%$ of the income distribution with housing costs exceeding $30 \%$ of their gross income), relationship status and children. Social support was constructed using the average of 10 items addressing aspects of emotional support (rated on a 7-point Likert scale),${ }^{18}$ categorised into tertiles. Wealth was defined as household assets minus debt and categorised into tertiles, recorded in 2014 as wealth questions were not asked in 2011. Lifestyle factors included alcohol consumption, smoking, physical activity and self-reported body mass index (BMI). Baseline mental health was included as a covariate. Specific categories of each variable are described in table 1 .

\section{Propensity score approach}

Propensity score methods provide an alternative method to adjust for confounding compared with traditional regression models and are particularly useful in analyses with many potential confounders. ${ }^{19} 20$ We estimated a propensity score for each individual by fitting a logistic regression model for disability acquisition including all covariates (potential confounders and predictors of mental health; see figure 1). ${ }^{21}$ We employed inverse probability weighting (IPW), which uses propensity scores to create a weight for each individual. ${ }^{22}$ The distribution of the weights was examined using boxplots (see online Supplementary file 1). Some very large weights resulted from people with disabilities with very low propensity scores; therefore, we trimmed weights at the 99 th percentile. $^{23}$

The average causal effect (ACE), which represents the mean causal effect for all individuals in the population, was estimated from a linear regression model with IPW as probability weights, using robust estimators given that the weights are estimated. ${ }^{24}$ To verify whether balance of the confounding variables was achieved, percentage standardised differences between the groups were calculated for each covariate, with differences less than $10 \%$ considered to reflect good balance. ${ }^{25}$

\section{Statistical analysis}

Descriptive analyses were conducted, summarising mental health for people who acquired a disability and those who did not, as well as demographic, socioeconomic
Table 1 Demographic, socioeconomic and mental health characteristics of people with and without disabilities, Australia, 2011-2014.

\begin{tabular}{lll}
$\frac{\text { Disability }}{n=311}$ & $\frac{\text { No disability }}{n=6150}$ \\
\cline { 3 - 3 }$n$ & $\frac{n}{n} \%$
\end{tabular}

\begin{tabular}{lllll}
\hline $\begin{array}{l}\text { Age (years) } \\
<30\end{array}$ & 43 & 13.8 & 1901 & 30.9 \\
\hline $30-44$ & 66 & 21.2 & 1957 & 31.8 \\
\hline $45-69$ & 101 & 32.5 & 1568 & 25.5 \\
\hline $60+$ & 101 & 32.5 & 724 & 11.8 \\
\hline $\begin{array}{l}\text { Sex } \\
\text { Men }\end{array}$ & 160 & 51.4 & 2928 & 47.6 \\
\hline $\begin{array}{l}\text { Women } \\
\text { Country of birth }\end{array}$ & 151 & 48.6 & 3222 & 52.4 \\
\hline $\begin{array}{l}\text { Australia } \\
\begin{array}{l}\text { Other } \\
\text { Education }\end{array}\end{array}$ & 241 & 77.5 & 4862 & 79.1 \\
$\begin{array}{l}\text { Bachelor or } \\
\text { higher }\end{array}$ & 70 & 22.5 & 1288 & 20.9 \\
$\begin{array}{l}\text { Secondary, } \\
\text { certificate, } \\
\text { diploma }\end{array}$ & 149 & 18.0 & 1820 & 29.6 \\
\hline
\end{tabular}

\begin{tabular}{llllrl}
$\begin{array}{l}\text { Did not complete } \\
\text { secondary }\end{array}$ & 106 & 34.1 & 1428 & 23.2 \\
\hline $\begin{array}{l}\text { Employment } \\
\text { Employed }\end{array}$ & 193 & 62.1 & 4810 & 78.2 \\
\hline $\begin{array}{l}\text { Unemployed } \\
\text { Not in the labour }\end{array}$ & 9 & 2.9 & 176 & 2.9 \\
\hline \begin{tabular}{l} 
force \\
\hline
\end{tabular} & & 35.0 & 1164 & 18.9 \\
\hline
\end{tabular}

Income

$\begin{array}{lllll}\text { Q5 (highest) } & 56 & 18.0 & 1662 & 27.0 \\ \text { Q4 } & 62 & 19.9 & 1495 & 24.3 \\ \text { Q3 } & 68 & 21.9 & 1252 & 20.4 \\ \text { Q2 } & 62 & 19.9 & 1170 & 19.0 \\ \text { Q1 (lowest) } & 63 & 20.3 & 571 & 9.3\end{array}$

Wealth

\begin{tabular}{lllll} 
High & 114 & 36.7 & 2497 & 40.6 \\
Medium & 106 & 34.1 & 2052 & 33.4 \\
Low & 91 & 29.3 & 1601 & 26.0 \\
\hline
\end{tabular}

Financial hardship

\begin{tabular}{lccrrr}
$\begin{array}{l}\text { Prosperous/very } \\
\text { comfortable }\end{array}$ & 39 & 12.5 & 1278 & 20.8 \\
$\begin{array}{l}\text { Reasonably } \\
\text { comfortable }\end{array}$ & 170 & 54.7 & 3386 & 55.1 \\
$\begin{array}{l}\text { Just getting by/ } \\
\text { very poor }\end{array}$ & 102 & 32.8 & 1486 & 24.2 \\
\hline
\end{tabular}

Housing tenure

$\begin{array}{lllll}\text { Outright owner } & 120 & 38.6 & 1773 & 28.8\end{array}$

Continued 
Table 1 Continued

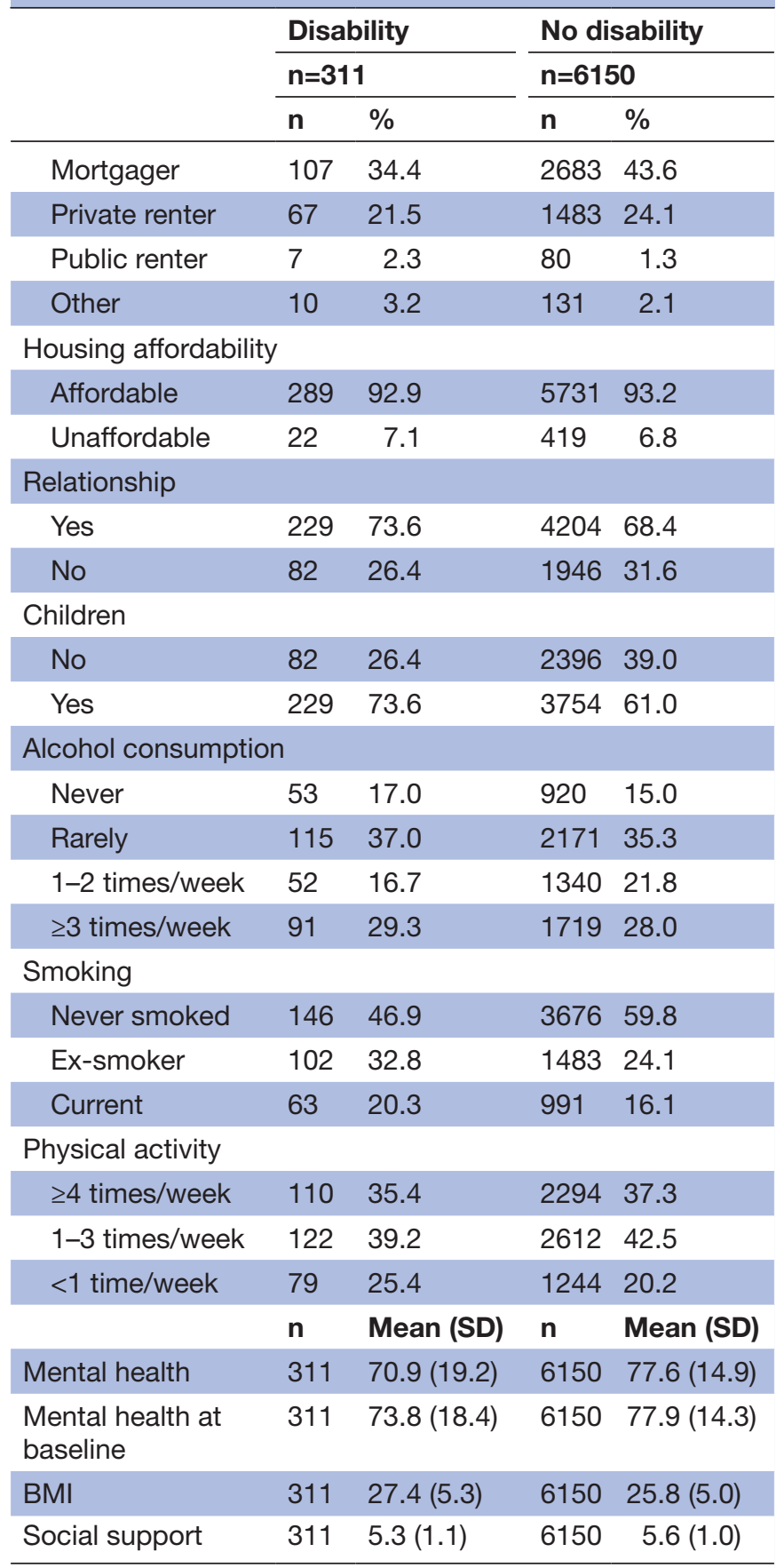

BMI, body mass index.

and mental health characteristics prior to disability acquisition.

The ACE of disability acquisition on mental health at the final wave was estimated using linear regression models for mental health with disability acquisition as the sole independent variable with IPW weights. We tested for additive effect modification by demographic and socioeconomic factors identified a priori that were thought to show evidence for effect heterogeneity based on substantive knowledge, including age, sex, country of birth, education, employment, income, wealth, financial hardship, housing tenure, housing affordability, relationship status and children. We quantified excess effects associated with each characteristic, which is the additional difference in mental health that exceeds the sum of each factor considered separately, and the effect of disability acquisition within each stratum of the effect modifier. We included an interaction term between disability acquisition and each effect modifier in turn, and tested for effect modification using likelihood ratio tests and Wald tests. Analyses were conducted using Stata/SE 12.1. ${ }^{26}$

\section{Missing data}

Investigation and handling of missing data was conducted for people who were eligible for inclusion in the sample; therefore, people who were lost to follow-up were excluded from the analyses. The distribution of baseline covariates was compared between those with and without missing observations. Missingness was associated with the values of measured variables, suggesting that the data were not missing completely at random. Therefore, multiple imputation using chained equations with 50 imputations was employed to maximise the validity of the findings as this approach assumes the data are missing at random, that is, systematic differences between missing and observed values are explained by differences in observed data. ${ }^{27}$ The multiple imputation model included all variables in the target analysis and additional auxiliary variables (further details in online Supplementary file 2).

\section{Sensitivity analyses}

A series of sensitivity analyses were conducted to test the robustness of findings. First, we repeated analyses using alternative propensity score approaches (stratification and covariate adjustment). Second, we conducted a complete case analysis. Third, we excluded people who acquired psychological impairments, as we would expect them to have poorer mental health associated with disability.

\section{RESULTS}

A total of 8323 individuals were eligible for inclusion in the analysis, 387 with disability and 7936 controls. Complete data were available for 6461 participants (78\%); figure 2 describes eligibility criteria and missing data. Data were missing for mental health, financial hardship, housing tenure and affordability, relationship status, social support, alcohol consumption, smoking, physical activity and BMI. People with missing data had poorer mental health and were more likely to experience socioeconomic disadvantage across all indicators (online Supplementary file 2).

There were differences in the distribution of covariates at baseline between people who acquired a disability and those who did not (table 1). People who acquired a disability were older and more likely to be male and born outside of Australia. They experienced greater socioeconomic disadvantage including low education, employment rates, income, wealth and social support, greater financial hardship, higher rates of smoking, less physical activity 
Potential effect modifiers

Age, sex, country of birth, education, employment, income, wealth, financial hardship, housing tenure \& affordability, relationship status, children

\section{Confounders}

Age, sex, country of birth,

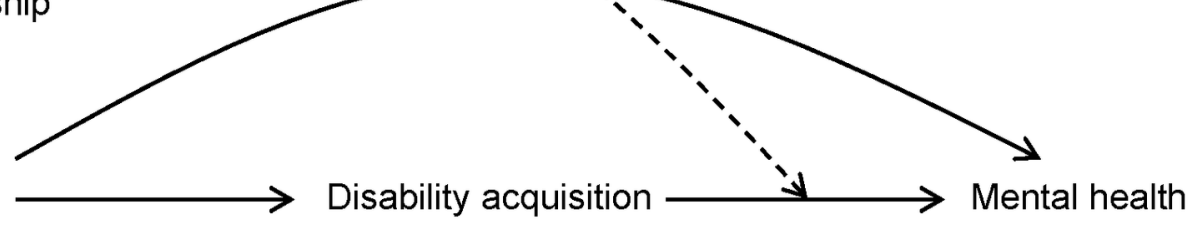
education, employment, income, wealth, financial hardship, housing tenure \& affordability, relationship status, children, smoking, alcohol consumption, physical activity, BMI

\& social support

$$
2011
$$

Figure 1 Causal diagram depicting potential confounders and effect modifiers of the association between disability acquisition and mental health.

and higher BMI, although they were similar with regard to housing tenure, affordability and alcohol consumption. Those with disabilities were more likely to be in a relationship and have children. The groups differed in terms of mental health, both before and after disability. At the final wave, those with disabilities had poorer mental health compared with those without (70.9 compared with 77.6). But even at baseline, those in the disability sample had poorer mental health compared with people who did not acquire a disability, with mean MHI scores of 73.8 compared with 77.9 (table 1).

These differences highlighted the need for a propensity score approach to ensure adequate control for confounding. The estimated propensity score model indicated that the strongest predictors of disability acquisition were age, education, unemployment, wealth, relationship status and mental health at baseline. The distribution of propensity scores differed between people with and without disabilities, but the IPW balanced the distribution between the groups (see online Supplementary file 3).

The IPW performed well at balancing the baseline covariates, with standardised differences between the groups much lower in the weighted sample. After IPW, the groups were comparable in terms of mental health at baseline, with MHI scores of 77.0 for people with disability and 77.7 for those without disability, a standardised difference of $4 \%$ compared with $25 \%$ in the unweighted sample. The groups also became more comparable in terms of all other baseline covariates, with standardised differences less than $10 \%$ for most variables, except age, children, smoking status and BMI, which were all under 17\% (figure 3 and online Supplementary file 4).

The ACE of disability acquisition on mental health was estimated to be a 5-point decline in MHI score (estimated mean difference: $-5.1,95 \%$ CI -7.2 to -3.0$)$. The joint effects of disability and socioeconomic characteristics were largest for people who acquired a disability who were public renters $(-23.2,95 \% \mathrm{CI}-33.8$ to -12.6$)$, unemployed $(-17.2,95 \%$ CI -30.7 to -3.7$)$, had low income $(-15.0,95 \%$ CI -20.9 to -9.1$)$, poor wealth $(-13.2,95 \%$ CI -17.3 to -9.1$)$, experienced financial hardship $(-12.5$, $85 \% \mathrm{CI}-16.1$ to -8.8$)$, were in unaffordable housing $(-13.1$, $95 \% \mathrm{CI}-22.0$ to -4.3$)$, and those in the younger age groups $(<30:-15.5,95 \%$ CI -20.0 to $-10.9 ; 30-44:-14.3,95 \%$ CI -18.9 to -9.8 ) (table 2 , column 1 ).

There was strong evidence from the tests for interaction of an excess effect between disability and income and disability and relationship status on mental health (table 2, column 4 ), with excess effects of more than five points for people who were not in a relationship $(-5.1,95 \%$ CI -9.8 to -0.4$)$ and more than 11 points for those in the lowest income category $(-11.4,95 \% \mathrm{CI}-18.5$ to -4.3 ) (table 2 , column 4 ). As such, the effects of disability on mental health were more detrimental among people with lower income than people with higher income (Q1: $-12.5,95 \%$ CI -18.5 to -6.5 ; Q5: $-1.1,95 \% \mathrm{CI}-4.9$ to 2.7$)$ and people who were not in a relationship $(-8.8,95 \% \mathrm{CI}-12.9$ to -4.8$)$ compared with those who were $(-3.7,95 \% \mathrm{CI}-6.1$ to -1.4$)$ (table 2 , column 2 ). There were also large excess effects for other socioeconomic characteristics, with particularly large effects for those unemployed, with low wealth, experiencing financial hardship, unaffordable housing and for public renters, suggesting that these characteristics also modified the effect of disability acquisition on mental health, although the interaction terms were not statistically significant (table 2 , column 5).

The first sensitivity analysis using alternative propensity score approaches did not materially change the results, suggesting that models with IPW were robust. In the second sensitivity analysis, the complete case analysis, despite only 


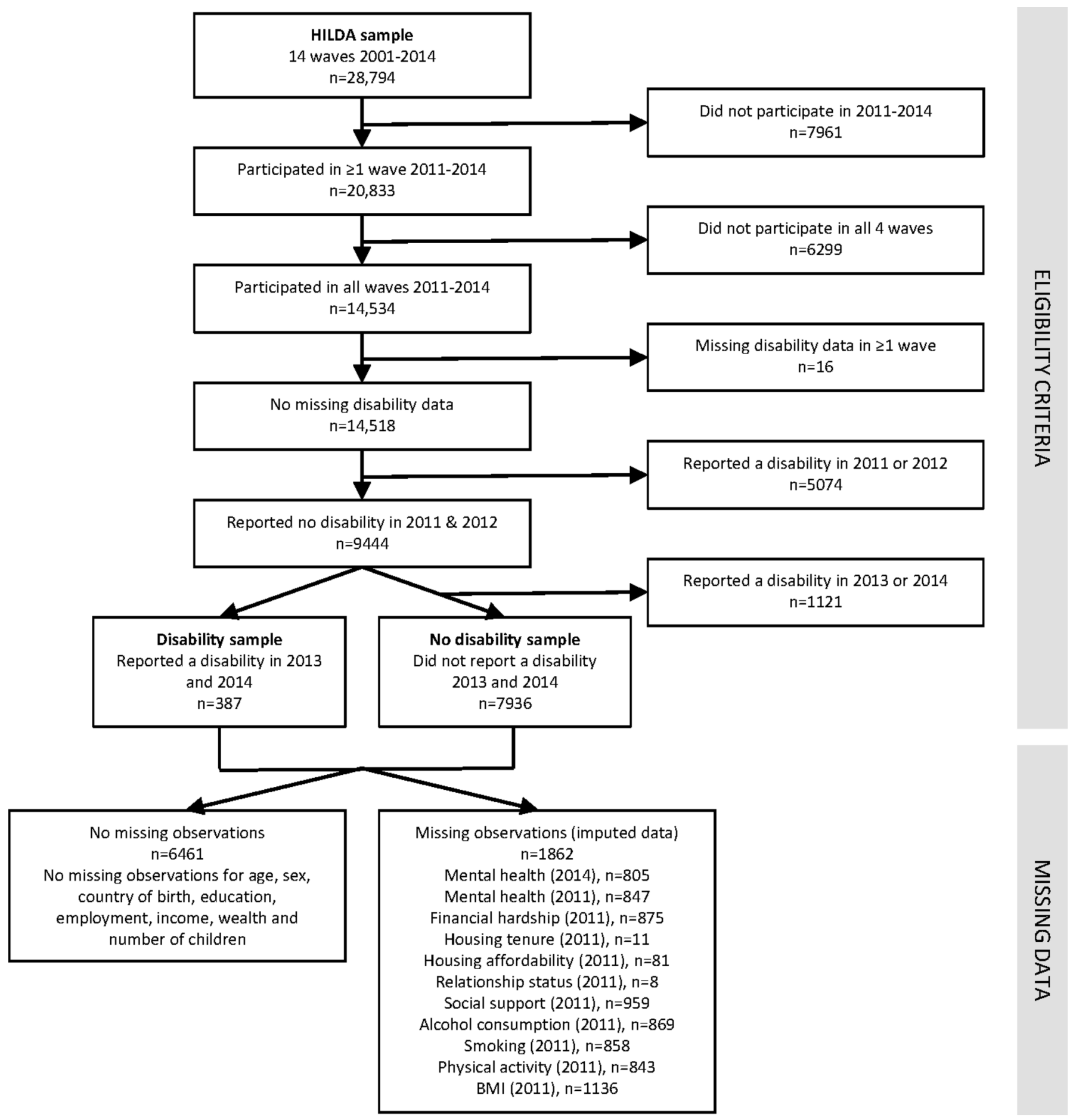

Figure 2 Flow diagram showing sample selection and missing data.

small changes in the magnitude of individual coefficients, the gradients in the excess effects across categories of socioeconomic characteristics were more pronounced, and as a result there was additional evidence for effect modification for education, wealth, housing tenure and housing affordability. The third sensitivity analysis excluding people with psychological impairments reduced the number of people acquiring a disability from 387 to 316 . For most covariates, the effect estimates between disability acquisition and mental health were slightly attenuated and the confidence intervals were wider (and no longer statistically significant), however the patterns of association were similar. A notable exception was the difference in the magnitude of effect according to relationship status, for which an excess effect was no longer apparent.

\section{DISCUSSION}

This is the first study to examine a broad range of socioeconomic and demographic characteristics to understand 


\section{Covariates}

Age: $<30$

30-44

45-59

$60+$

Sex: men

women

COB: Australia

Other

Education: $\geq$ bachelor

secondary

not secondary

Employment: employed

unemployed

NILF

Income: Q5 (highest)

Q4

Q3

Q2

Q1 (lowest)

Wealth: high

medium

low

Financial hardship: prosperous medium poor

Housing tenure: owner

mortgager

private rental

public rental

other

Housing affordability: affordable unaffordable

Relationship: yes

no

Children: no

yes

Social support

Alcohol consumption: never

rarely

1-2/week

$\geq 3$ /week

Smoking: never

ex-smoker

current

Physical activity: $\geq 4 /$ week

1-3/week

$<1 /$ week

BMI

Mental health

Mental health at baseline

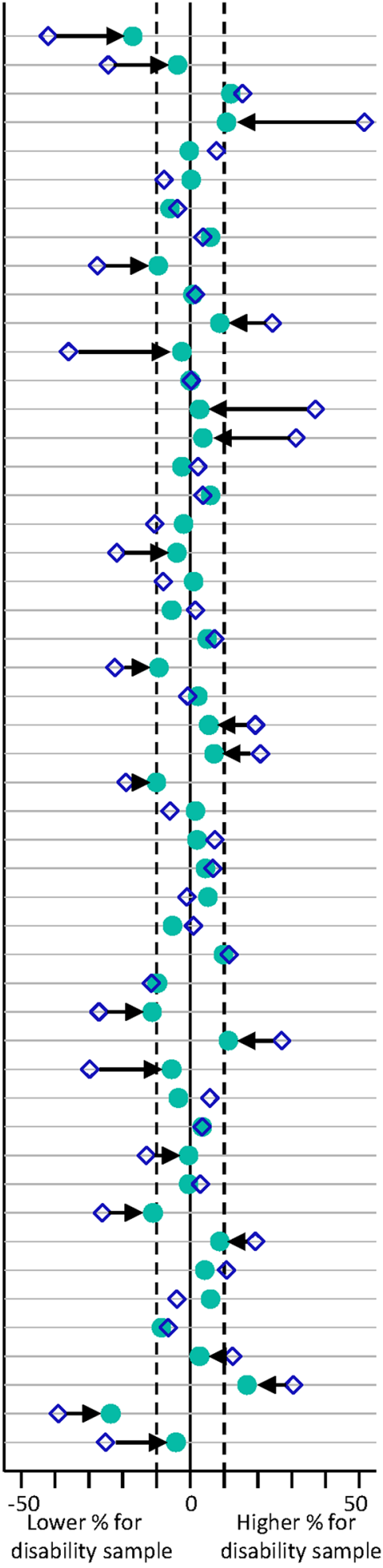

Percentage standardised difference

Figure 3 Percentage standardised differences between people with and without disabilities for each covariate before and after IPW on the propensity score (unweighted sample: $\diamond$ I IPW-weighted sample: $)$ ); the dashed lines indicate the $10 \%$ differences which reflect good balance of confounders.

the relative importance of socioeconomic influences on the effect of disability acquisition in adulthood on mental health. Our finding that disability interacts with socioeconomic characteristics to shape mental health underlines the importance of considering intersectionality in studies of disability and health inequalities. ${ }^{14}$ There was strong evidence that the effect was greater for people on low income and those not in a relationship, and there were 

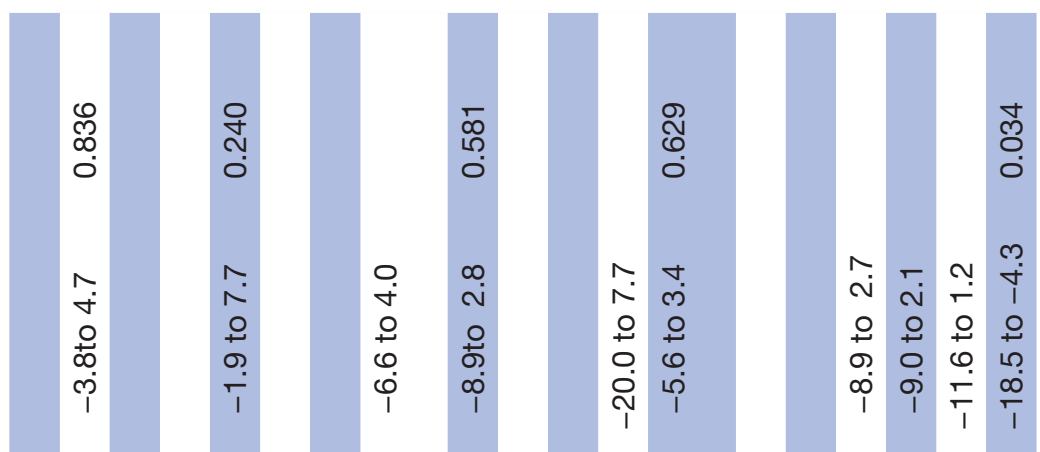

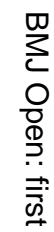

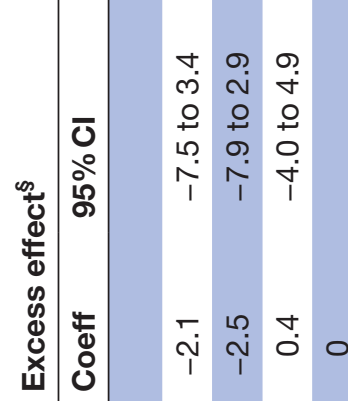

$\hat{\dot{\tau}}$

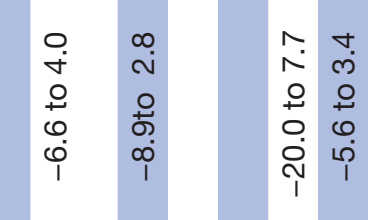

芦

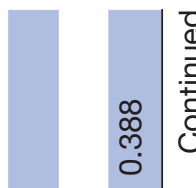

ก ก

ช กั

우우

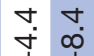

$\vec{\circ}$

$\overrightarrow{\vec{\omega}}$

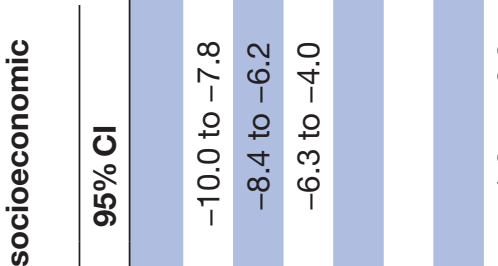

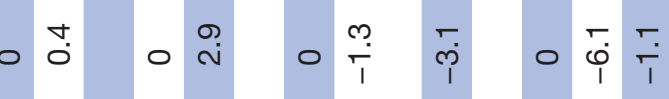

○

$\begin{array}{lll}m & 0 \\ 1 & 0 \\ 1 & 0 \\ 0 & 0\end{array}$

गे

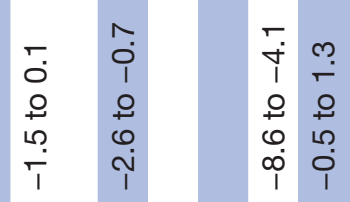

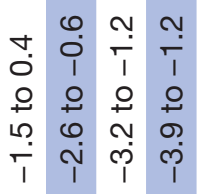

م)

웅

우

๑) ต

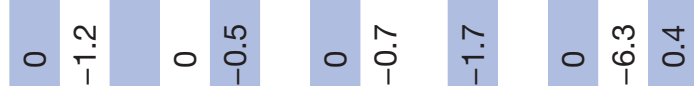

0 م

ᄃ

ஸ

ก ก

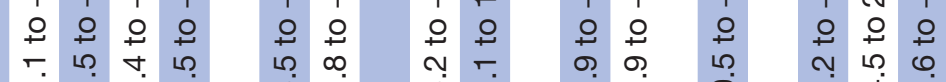

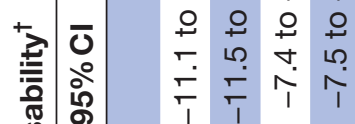

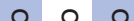

1

ब

î

๑ 두

ம.

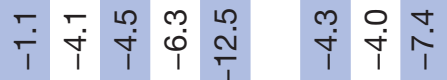

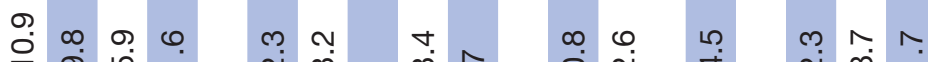

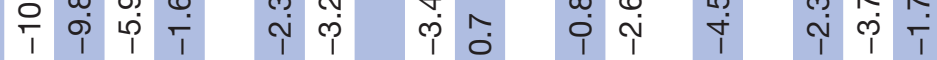
ヘิ

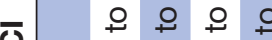
+2 웅

$+\circ$

+2 우운

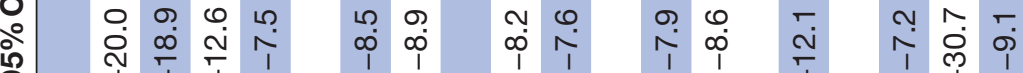




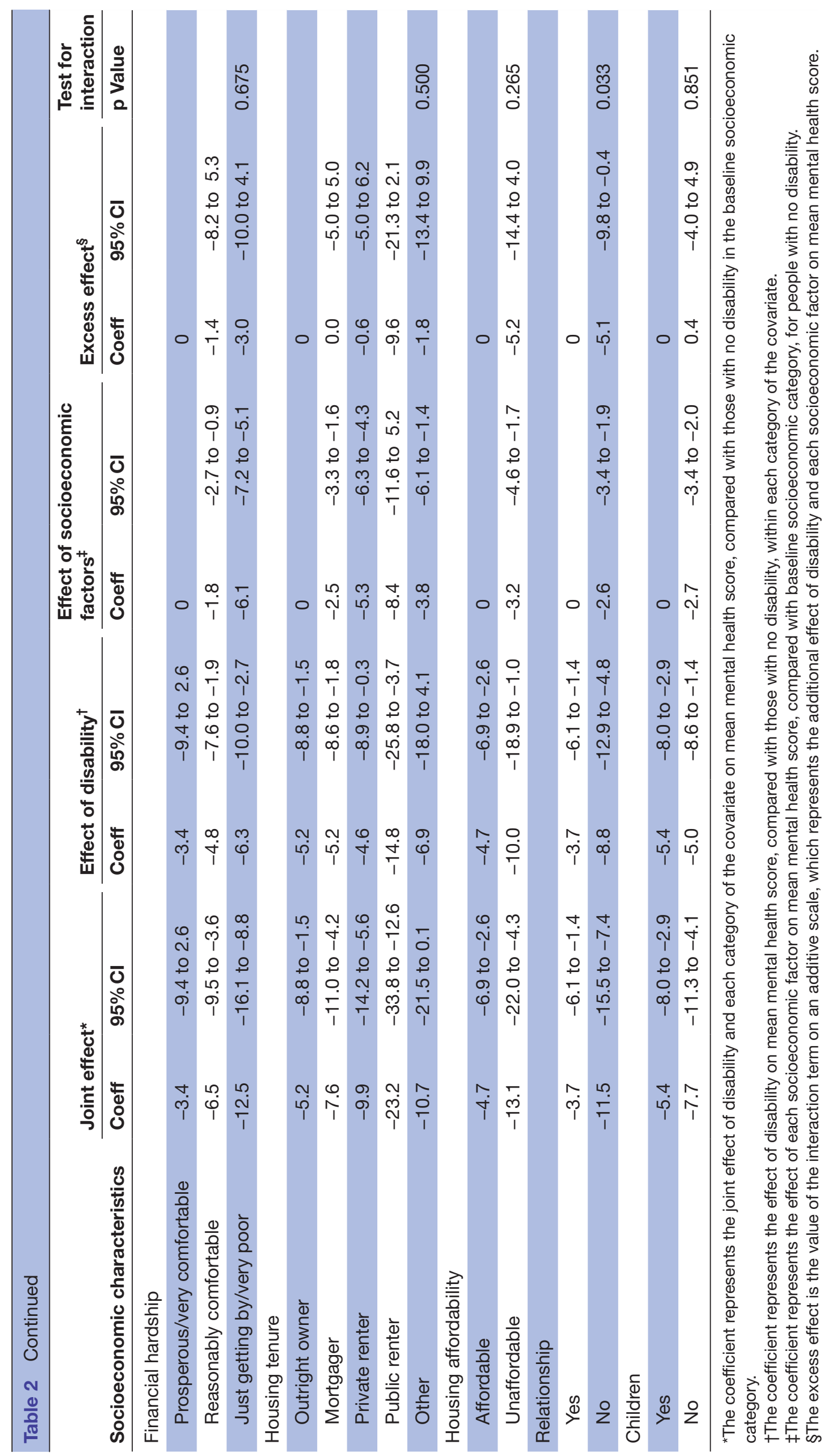


also large differences in the magnitude of the effect according to employment status, wealth, financial hardship, housing tenure and housing affordability, although the interaction terms were not statistically significant. The results were similar to other studies, finding some evidence of differences in the effect by wealth, ${ }^{36}$ housing tenure and affordability, ${ }^{2}$ although effect modification by education was less pronounced than in other studies. ${ }^{5}$

In this study, people who acquired a disability experienced on average a 5-point decline in mental health, substantially exceeding a 3-point difference considered to represent a clinically meaningful change. ${ }^{28}$ Importantly, the results suggest that the mental health effects are heterogeneous, with some people experiencing much larger mental health declines, for example, a 12.5-point decline for those in the lowest income quintile.

This study benefited from a number of strengths. The longitudinal nature of the data enabled identification of incident cases of disability and characteristics prior to disability. We used IPW weighting to control for confounding, to ensure that the groups were comparable prior to disability acquisition, strengthening our ability to interpret estimates as causal effects of disability on mental health. We trimmed weights at the 99th percentile to avoid large weights leading to inflated standard errors, resulting in poorer balance between the groups, with standardised differences of four covariates ranging from $10 \%$ to $17 \%$. While this is a limitation, we believed this was preferable to extreme weights. Finally, results from other propensity score approaches including covariate adjustment and stratification were similar, suggesting that the IPW models were robust.

Our analysis has a number of limitations. We tested for effect modification across many variables, which increased the risk of type I error. However, the variables were selected a priori and all associations were in the expected direction, therefore unlikely to have arisen by chance. In addition, only two of the variables showed statistical evidence of effect modification, despite large differences in the magnitude of the effect estimates. Although we had a large sample and a continuous outcome, the power to detect interactions was low because only $5 \%$ of study participants acquired a disability. Missing data may have introduced selection bias. The complete case analysis suggested effect modification by a greater number of socioeconomic characteristics compared with results using multiple imputation. This was explained by the relatively poorer mental health of disabled individuals with high socioeconomic status who had missing data compared with those with complete data, which led to an overestimation of gradients in the complete case analysis. However, multiple imputation is likely to have corrected for this selection bias because the method requires the less stringent assumption of missing at random (ie, missingness depends on measured covariates as observed in our study), whereas the complete case analysis assumes the participants with missing data represent a random sample of those that were intended to be observed (missing completely at random). We did not account for survey weights, which may affect estimated standard errors; however, adjustment for the survey weights in conventional linear regression models did not substantially change the results. Finally, there was potential for dependent misclassification bias, where misclassification of the outcome depends on misclassification of the exposure because both disability and mental health were self-reported.

Although the patterns of association were generally similar, there were some differences in the results of the sensitivity analysis excluding people with psychological impairments, particularly the attenuation of effect modification by relationship status. One assumption underpinning the propensity score approach is the no-multiple-versions-of-treatment assumption, which stipulates that the potential outcomes under each level of the exposure are well defined and take on a single value. ${ }^{29}$ It is possible that the effect of disability on mental health may vary according to the 'version' of the exposure, such as different types or severity of disability, however we lacked power to examine differences by disability characteristics. Therefore, estimated effects should be interpreted as average causal effects of disability on mental health, which may affect the transportability of results as the estimated effect depends on the distribution of disability characteristics in the sample. ${ }^{30}$ People with severe disabilities are less likely to participate in HILDA, therefore our results are likely to underestimate the population effect of disability acquisition on mental health.

The finding that the effect of disability acquisition on mental health depends on people's socioeconomic characteristics has important implications for disability, social and public health policies. People who are socioeconomically disadvantaged (particularly those with low income and not in a relationship) are a vulnerable subgroup of people with disabilities who are likely to experience greater mental health effects, and therefore may benefit most from targeted social and health policies interventions. Therefore, addressing the social determinants of health of people with disabilities, such as interventions that improve people's financial situation, for example investing in employment and education services for people with disabilities, may have substantial beneficial impacts on mental health. Such interventions have implications for the mental health and well-being of people with disabilities and their families, as well as for longterm health and welfare costs. It is also important that high-quality mental health services are accessible and affordable for people who acquire a disability particularly if they are socioeconomically disadvantaged or not in a relationship.

Acknowledgements This article uses unit record data from the Household, Income and Labour Dynamics in Australia (HILDA) Survey. The HILDA Project was initiated and is funded by the Australian Government Department of Social Services (DSS) and is managed by the Melbourne Institute of Applied Economic and Social Research (Melbourne Institute). The findings and views reported in this paper, however, are those of the author and should not be attributed to either DSS or the Melbourne Institute. 
Contributors ZA conceived the research question, conducted the analysis, interpreted the results and prepared the manuscript. JAS, RB and AMK made substantial contributions to the study conception, the data analysis methods, the interpretation of results and critically reviewed the manuscript. All authors read and approved the final manuscript.

Funding This work was supported by an Australian Government Research Training Program Scholarship and a National Health and Medical Research Council Postgraduate Scholarship (1093740) to ZA.

Competing interests None declared.

Patient consent We have only used de-identified data.

Ethics approval Ethics approval for this study was provided by the Human Ethics Advisory Groups at the Melbourne School of Population and Global Health at the University of Melbourne.

Provenance and peer review Not commissioned; externally peer reviewed.

Data sharing statement The HILDA Survey data can be obtained under strict licensing arrangements, subject to approval by the Australian Government Department of Social Services. Further details are available at: https://www. melbourneinstitute.com/hilda/data/data_ind.html.

Open Access This is an Open Access article distributed in accordance with the Creative Commons Attribution Non Commercial (CC BY-NC 4.0) license, which permits others to distribute, remix, adapt, build upon this work non-commercially, and license their derivative works on different terms, provided the original work is properly cited and the use is non-commercial. See: http://creativecommons.org/ licenses/by-nc/4.0/

(c) Article author(s) (or their employer(s) unless otherwise stated in the text of the article) 2017. All rights reserved. No commercial use is permitted unless otherwise expressly granted.

\section{REFERENCES}

1. ABS. Disability, ageing and carers: summary of findings 2012 (4430.0): ABS, 2013

2. Kavanagh AM, Aitken Z, Baker E, et al. Housing tenure and affordability and mental health following disability acquisition in adulthood. Soc Sci Med 2016;151:225-32.

3. Kavanagh AM, Aitken Z, Krnjacki L, et al. Mental Health following acquisition of disability in Adulthood--the impact of wealth. PLoS One 2015;10:e0139708.

4. AlHW. Health of Australians with disability: health status and risk factors. Bulletin 83. Canberra: : AlHW, 2010. Cat no. AUS 132.

5. Mandemakers JJ, Monden CW. Does education buffer the impact of disability on psychological distress? Soc Sci Med 2010;71:288-97.

6. Smith DM, Langa KM, Kabeto MU, et al. Health, wealth, and happiness: financial resources buffer subjective well-being after the onset of a disability. Psychol Sci 2005;16:663-6.

7. Yang Y. How does functional disability affect depressive symptoms in late life? the role of perceived social support and psychological resources. J Health Soc Behav 2006;47:355-72.

8. Kariuki M, Honey A, Emerson E, et al. Mental health trajectories of young people after disability onset. Disabil Health J 2011;4:91-101.

9. Emerson E, Kariuki M, Honey A, et al. Becoming disabled: the association between disability onset in younger adults and subsequent changes in productive engagement, social support, financial hardship and subjective wellbeing. Disabil Health $\mathrm{J}$ 2014;7:448-56.

10. Yang Y, George LK. Functional disability, disability transitions, and depressive symptoms in late life. J Aging Health 2005;17:263-92.

11. Honey A, Emerson E, Llewellyn G. The mental health of young people with disabilities: impact of social conditions. Soc Psychiatry Psychiatr Epidemiol 2011;46:1-10.

12. Lynch J, Smith GD, Harper S, et al. Is income inequality a determinant of population health? Part 1. A systematic review. Milbank Q 2004:82:5-99.

13. Kapilashrami A, Hill S, Meer N. What can health inequalities researchers learn from an intersectionality perspective? understanding social dynamics with an inter-categorical approach? Social Theory \& Health 2015;13(3-4):288-307.

14. Bauer GR. Incorporating intersectionality theory into population health research methodology: challenges and the potential to advance health equity. Soc Sci Med 2014;110:10-17.

15. Mullings L, Schulz A. Intersectionality and health: an introduction. In: Mullings L, Schulz A, eds. Gender, race, class, and health: an intersectional approach. San Francisco: John Wiley \& Sons, 2006.

16. Summerfield M, Freidin S, Hahn M, et al. HILDA user Manual Release 12. 12 edn: Melbourne Institute of Applied Economic and Social research, 2013.

17. Butterworth P, Crosier T. The validity of the SF-36 in an Australian National Household Survey: demonstrating the applicability of the Household Income and Labour Dynamics in Australia (HILDA) Survey to examination of health inequalities. BMC Public Health 2004;4:44

18. Milner A, Krnjacki L, Butterworth P, et al. The role of social support in protecting mental health when employed and unemployed: a longitudinal fixed-effects analysis using 12 annual waves of the HILDA cohort. Soc Sci Med 2016;153:20-6.

19. Cousens S, Hargreaves J, Bonell C, et al. Alternatives to randomisation in the evaluation of public-health interventions: statistical analysis and causal inference. J Epidemiol Community Health 2011;65:576-81.

20. Williamson E, Morley R, Lucas A, et al. Propensity scores: from naive enthusiasm to intuitive understanding. Stat Methods Med Res 2012;21:273-93.

21. Brookhart MA, Schneeweiss S, Rothman KJ, et al. Variable selection for propensity score models. Am J Epidemiol 2006;163:1149-56.

22. Hernán MA, Robins JM. Estimating causal effects from epidemiological data. J Epidemiol Community Health 2006;60:578-86

23. Lee BK, Lessler J, Stuart EA. Weight trimming and propensity score weighting. PLoS One 2011;6:e18174.

24. Austin PC, Stuart EA. Moving towards best practice when using inverse probability of treatment weighting (IPTW) using the propensity score to estimate causal treatment effects in observational studies. Stat Med 2015;34:3661-79.

25. Williamson EJ, Forbes A. Introduction to propensity scores Respirology 2014;19:625-35.

26. Stata Statistical Software: release 12[program]. College Station, TX: StataCorp LP, 2011.

27. Sterne JA, White IR, Carlin JB, et al. Multiple imputation for missing data in epidemiological and clinical research: potential and pitfalls. BMJ 2009;338:b2393.

28. Ware JE, Kosinski M, Gandek B. SF-36 health survey: manual \& interpretation guide: Lincoln, RI:QualityMetric, 2000.

29. VanderWeele TJ, Hernán MA. Causal Inference under multiple versions of treatment. J Causal Inference 2013;1:1-20.

30. Hernán MA, VanderWeele TJ. Compound treatments and transportability of causal inference. Epidemiology 2011;22:368-77. 